(C) 1998 International Press

Adv. Theor. Math. Phys. 2 (1998) 987-1009

\title{
Duality without supersymmetry
}

\author{
Paul Fendley \\ Physics Department \\ University of Virginia \\ Charlottesville, VA 22901
}

\begin{abstract}
I show that physical quantities in several two-dimensional condensedmatter models are related to the Seiberg-Witten calculation of exact quantities in supersymmetric gauge theory. In particular, the magnetization in the Kondo problem and the current in the boundary sine-Gordon model can each be expressed in the form $\int d x / y$, where for example in the latter $y^{2}=x+x^{g}-u^{2}$ with $u$ related to the boundary mass scale (the analog of $\left.\Lambda_{\mathrm{QCD}}\right)$ and $g \propto R^{2}$, where $R$ is the radius of the boson. Thus for irrational $g$, the hyperelliptic curve $y(x)$ is of infinite genus, while for rational $g$ it is of finite genus. The models are integrable and possess a quantum-group symmetry for any $g$, but are supersymmetric only at $g=2 / 3$. Both models also possess unique forms of $g \rightarrow 1 / g$ duality.
\end{abstract}

\section{Introduction}

Several years ago, Seiberg and Witten showed that "duality" can imply a much more elaborate structure than merely invariance under inverted couplings. In $N=2$ supersymmetric gauge theories, they showed that one can exploit the analyticity implied by the supersymmetry in order to do nonperturbative computations [1]. This work has been generalized in many ways, but always to field theories with supersymmetry, since analyticity in some parameter is required. However, there seems to be no fundamental reason why supersymmetry is required for such analyticity to be present. In this paper, I will show that several fundamental models of integrable

e-print archive: http://xxx.lanl.gov/abs/hep-th/9804108 
1+1-dimensional field theory which are not supersymmetric exhibit similar behavior.

A familiar fact from complex analysis is that if one knows the poles, residues and asymptotic behavior of a function, then that function can be reconstructed uniquely if it is analytic except at the poles. Similarly, if a function has branch-point singularities but is analytic elsewhere, one can reconstruct the complete function by knowing enough about the behavior at the singularities. In the simplest non-trivial cases, the function $f(u)$ can be expressed in terms of integrals like

$$
f(u)=\int_{\mathcal{C}} \frac{d x}{y}
$$

for some contour $\mathcal{C}$. Here $y^{2}$ is a cubic polynomial in $x$ with coefficients depending on $u$; by redefining $x$ one can always write it in the form

$$
y^{2}=x^{3}+a(u) x+b(u) .
$$

The integrand has three square-root branch points at the roots of this polynomial, so there are two different branch cuts in the $x$ plane, one connecting two of the branch points, and the other connecting the third to infinity. The sign ambiguity in $y$ can be fixed by allowing $x$ to take values on two sheets. Including the point at infinity, each sheet is a sphere with two branch cuts. The two spheres must be glued together along both branch cuts; this is equivalent to a torus. For higher-order polynomials in $x$, there are more singularities and the corresponding surface has higher genus. At special values of $a$ and $b$ (i.e. special values of $u$ ) where two of these roots coincide, the integral (1) logarithmically diverges if the contour goes in between these two roots. These values of $u$ are the singularities of $f(u)$, and are values of $u$ where one of the cycles of the surface is pinched to a point.

In [1], Seiberg and Witten showed that certain quantities in $N=2$ supersymmetric $S U(2)$ gauge theory are holomorphic functions of $u$, an order parameter related to the vacuum expectation value of the Higgs field. The "duality" is the fact that the monodromies around the singularities in the $u$ plane are given by $S L(2, Z)$ transformations generalizing $g \rightarrow 1 / g$. These monodromies can exactly be determined by perturbation theory and an exact mass formula for certain kinds of particles called BPS states. Thus various physical quantities (for example the effective coupling constant, the beta function and the mass gap) are given exactly and non-perturbatively by integrals like (1). Their work has now been generalized to many supersymmetric gauge theories, and also is deeply related to duality in string theory. There are many review articles on these subjects; see for example [2]. 
In integrable models, it has long been known how to exploit analyticity to derive various physical quantities from information like functional relations. In this paper I will show that physical quantities in several integrable models of 1+1-dimensional boundary quantum field theory can be expressed as integrals like (1). The theories, the Kondo model and the boundary sineGordon model, are not supersymmetric, and it is not likely that there is a hidden supersymmetry. Here the analyticity seems to follow from the integrability or, equivalently, the quantum-group symmetry of the models. The distinction between the models discussed here and integrable models where analyticity has previously been utilized is that these models obey intriguing, and I believe unique, forms of $g \rightarrow 1 / g$ duality $[3,4]$. This sort of duality, which generalizes the electric-magnetic duality of electromagnetism, was observed long ago in some gauge theories [5], providing one of the motivations for the work of Seiberg and Witten. This paper thus explores two somewhat different forms of duality: the ability to express physical quantities as curves around branch-point singularities, and the ability to relate different physics by a $g \rightarrow 1 / g$ mapping.

While the main purpose of this paper is to discuss the Kondo and boundary-sine Gordon models as surprising manifestations of Seiberg-Witten theory, it is worthwhile noting that both are extremely interesting in their own right. Not only are they fundamental models of strongly-interacting statistical mechanics, but both have been observed experimentally. In particular, the Kondo model used successfully for decades to describe dilute magnetic impurities in a conductor (see e.g. $[6,7]$ ) while the boundary sine-Gordon model describes the current-carrying edge excitations tunneling through a point contact in a fractional quantum Hall device (see [8]). The duality in the latter maps Laughlin quasiparticles to electrons.

In section 2 I introduce the Kondo model and recall various important results from the Bethe ansatz computations. Section 3 contains the main result of the paper, the writing of the magnetization in the Kondo problem as an integral over a cycle of a curve. This form is equivalent to the Bethe ansatz result, but much simpler and more useful. For example, it gives the entire singularity structure of the magnetization. Section 4 discusses a peculiar duality present in the Kondo and boundary sine-Gordon models. Section 5 highlights the $S U(2)$ invariant limit of the Kondo problem, which physically is the most reminiscent of QCD. 


\section{The Ingredients}

In this section I introduce the Kondo model, and discuss the ingredients necessary for understanding its type of Seiberg-Witten duality.

The Kondo model is one of the fundamental models of statistical mechanics. It describes three-dimensional non-relativistic electrons coupled to a single impurity spin. Writing the electron wavefunction in terms of spherical harmonics around the impurity, the most relevant interaction comes from the $s$-waves. Since these are spherically symmetric, the results depend only on the radial coordinate, and the problem is effectively $1+1$ dimensional: space is a half-line, with the impurity located at the boundary. Moreover, in the one-dimensional effective model, the fermions are relativistic and massless because one is concerned only with excitations near the Fermi surface. When $p$ is the momentum difference from the Fermi momentum $p_{F}$ and $E$ the energy difference from the Fermi energy $p_{F}^{2} / 2 m$, $E+E_{F}=\left(p+p_{F}\right)^{2} / 2 m \approx p_{F}^{2} / 2 m+v_{F} p$. Therefore the relativistic dispersion relation $E=p v_{F}$ holds, with the Fermi velocity $v_{F}=p_{F} / m$ playing the role of the speed of light. Henceforth $v_{F}=1$. Since there are two spins of electrons in the original three-dimensional problem, there are two flavors of fermions in the effective one-dimensional problem. One can then form an $S U(2)$ "spin" current $\mathbf{J}=\psi^{\dagger i} \sigma_{i j} \psi_{j}$ using the Pauli matrices $\sigma$, and the $U(1)$ "charge" current $\mathcal{J}=\psi^{\dagger i} \psi_{i}$. In conformal field theory language, these yield $S U(2)_{1}$ and $U(1)$ WZW theories respectively, each with a central charge $c=1[9]$.

The impurity is represented by a quantum-mechanical spin $\mathbf{S}$ in the spin$S$ representation. An impurity located at $x=0$ is coupled antiferromagnetically to the fermions by a term in the Lagrangian $-\lambda \mathbf{J}(0) \cdot \mathbf{S}$ for positive $\lambda$. There is no integral in this term because the interaction takes place at a single point in space, the boundary of the half-line. The coupling $\lambda$ is dimensionless since the current is of dimension one, so naively it seems that this coupling should be marginal and preserve conformal symmetry at the boundary. However, Kondo observed more than three decades ago that if one does perturbation theory in $\lambda$, there is a short-distance divergence [10]. Thus the interaction term is relevant, and a mass scale is present in the theory. In particle-physics language, the Kondo model is asymptotically free and undergoes dimensional transmutation. This scale generated is usually called the Kondo temperature $T_{K}$, and it is completely analogous to $\Lambda_{Q C D}$ in gauge theory. In terms of the original parameter $\lambda$,

$$
T_{K} \sim \lambda^{1 / 2} e^{- \text {const } / \lambda} .
$$

The precise relation as well as the positive constant are not universal (i.e. 
depend on regularization procedure); we will discuss physics in terms of $T_{K}$ and will not need the detailed definition.

As $\lambda$ gets large (or more precisely, we study physics at energy scales below $T_{K}$ ), the system crosses over to a strongly-coupled phase. At $T_{K} \rightarrow \infty$, there is another fixed point, where the spin is screened by one of the electrons (because of Pauli exclusion only a single electron can bind to the impurity). At this strongly-coupled fixed point, the spin of the impurity is effectively reduced from $S$ to $S-1 / 2$. The physical quantity we will study is the magnetization $\mathcal{M}_{S}$ of the spin- $S$ impurity as a function of applied magnetic field $H$. At zero temperature, the $\mathcal{M}_{S}$ is a function only of the dimensionless quantity $H / T_{K}$, so $\mathcal{M}_{S}(\infty)=S$, while $M(0)=S-1 / 2$.

Since Kondo's work, the Kondo model has been analyzed using many different techniques. It was one of the first models to be thoroughly understood using scaling and renormalization-group techniques. Various properties of the strongly-coupled fixed point were understood using scaling arguments [11]. The crossover between the two fixed points was described using numerical renormalization-group techniques [12]. Subsequently, many quantities were computed exactly using the Bethe ansatz; for reviews see $[6,7]$. The exact result for the magnetization curves at zero temperature can be written in the form [3]

$$
\begin{aligned}
\mathcal{M}_{S}\left(\frac{H}{T_{K}}\right)= & S-\frac{1}{2}+\frac{i}{8 \sqrt{\pi}} \int_{-\infty}^{\infty} d \omega e^{-2 i \omega \ln \left(H / T_{K}\right)} \frac{\Gamma(1 / 2+i \omega)}{\omega+i \epsilon} \\
& \times\left[f_{+}(\omega)\right]^{2 S}\left[f_{-}(\omega)\right]^{2 S-1}
\end{aligned}
$$

where

$$
f_{\mp}(\omega) \equiv\left[\frac{ \pm i \omega+\epsilon}{2 \pi}\right]^{ \pm i \omega}
$$

with $\epsilon$ positive and tending to zero. The purpose of this paper is to write an equivalent expression for $\mathcal{M}_{S}$ which not only simplifies matters but which also yields a connection to the gauge-theory results of Seiberg and Witten.

I will discuss a more general model, the anisotropic Kondo model, which allows for $S U(2)$-breaking interactions with the impurity, namely

$$
\lambda \mathbf{J} \cdot \mathbf{S}+\lambda_{z} J_{z} S_{z}
$$

The model with anisotropy arises in dissipative quantum mechanics, where it describes a particle moving in a double well with dissipation [13]. A convenient way of parameterizing the anisotropy is given by bosonizing the model; in fact the Kondo model was one of the first models to be bosonized [15]. The $U(1)$ charge current $\mathcal{J}$ does not couple to the impurity, so it 
can be ignored. The bulk theory is described by a single free boson, with Lagrangian taking the form

$$
L_{0}=\frac{1}{4 \pi g} \int_{0}^{\infty} d x\left(\partial_{\mu} \phi\right)^{2}-\frac{H}{2 \pi g} \int_{0}^{\infty} d x \partial_{t} \phi .
$$

In the usual conventions of conformal field theory, the boson takes values on a circle so that $\phi=\phi+2 \pi R$. Changing the coupling $g$ is equivalent to rescaling the boson and changing the radius of this circle; i.e. $g \propto R^{2}$. In this paper it will be convenient to stick with the coupling $g$. In the bosonized language, the coupling to the impurity is then

$$
L_{S}=\lambda_{g}\left(S_{+} e^{-i \phi(0)}+S_{-} e^{+i \phi(0)}\right)-H S_{z} .
$$

The Lagrangian $L_{0}+L_{S}$ describes the Kondo model when $g \leq 1$. The anisotropy is parametrized by $g$, which is defined so that the isotropic case has $g=1$. One well-studied value (known as the Toulouse limit) is $g=1 / 2$, where the problem can be mapped on to one of free fermions. The magnetic field $H$ couples to the conserved $z$-component $S_{z}+\int J_{z}$ of the spin. The operators $\exp ( \pm i \phi(0))$ have boundary scaling dimension 1 when $g=1$ as they should; in general they have dimension $g$. Therefore

$$
T_{K} \propto \lambda_{g}^{1 /(1-g)}
$$

for $g \neq 1$. For a microscopic definition of $T_{K}$ (i.e. including the cutoff), see $[6]$.

Traditionally in the Kondo problem, one takes the matrices $S_{i}$ to act in the spin- $S$ representation of $S U(2)$. However, a subtlety arises for $g<$ 1 when spin is greater than $1 / 2$. For the problem to be integrable, one must instead take the matrices to act in the spin- $S$ representation of the quantum-group algebra $S U(2)_{q}$ instead of the ordinary $S U(2)$ algebra [16]. The quantum group is actually an algebra defined by the relations

$$
\left[S_{z}, S_{ \pm}\right]= \pm 2 S_{ \pm}, \quad\left[S_{+}, S_{-}\right]=\frac{q^{S_{z}}-q^{-S_{z}}}{q-q^{-1}}
$$

where $q=e^{i \pi g}$. In the isotropic case $q=-1$ or in the classical limit $q=1$, this reduces to the usual $S U(2)$ algebra. The Pauli matrices satisfy this algebra for any $q$, so the distinction between $S U(2)$ and $S U(2)_{q}$ is not important for $S=1 / 2$. In the following, the anisotropic Kondo model of spin- $S$ is defined to be the model with the $q$-deformed algebra; it can be identified with the "physical" Kondo model for $S=1 / 2$ or for $g=1$. 
The magnetization for spin- $1 / 2$ has been found exactly using the Bethe ansatz method. The dimensionless parameter $u$ is defined by

$$
u \equiv \frac{\Gamma\left(\frac{1}{2}+\frac{1}{2(1-g)}\right)}{2 \sqrt{\pi} \Gamma\left(1+\frac{1}{2(1-g)}\right)} \frac{H}{T_{K}} .
$$

The reason for the extra numerical factors in front is to conform with the usual definition of $T_{K}$ [6]. The model is "physical" when $H / T_{K}$ or equivalently $u$ is real and positive, but for reasons which will soon become apparent, we will study the magnetization for all values of $u$ in the complex plane. The Bethe ansatz result for the magnetization is [14]

$$
M_{1 / 2}(u)=\frac{i}{4 \pi^{3 / 2}} \int_{-\infty}^{\infty} d \omega e^{-2 i \omega \ln (u)} \frac{\Gamma(1 / 2+i \omega) \Gamma\left(1-\frac{i \omega}{1-g}\right)}{(\omega+i \epsilon) \Gamma\left(1-\frac{i g \omega}{1-g}\right)}
$$

In the limit $g \rightarrow 1, M_{1 / 2}$ here indeed reduces to the expression $\mathcal{M}_{1 / 2}$ in (3).

One can also derive a similar integral expression for $M_{S}(u)$ for any $g$, finding for example that although $M_{S}(\infty)=S$ as with the isotropic case, $M_{S}(0)=(S-1 / 2) / g$. Instead of using the integral expressions for higher $S$, it will be much more useful to utilize fusion relations. Fusion relations are a way of obtaining a new integrable model from an existing one. For example, from the spin-1/2 Heisenberg spin chain, one can obtain the integrable spin-1 chain by fusing together two neighboring spins [17]. In the Kondo model, this procedure allows one to construct the higher-spin impurity models by continuing the spin- $1 / 2$ model to imaginary couplings. The partition functions $Z_{S}\left(H / T_{K}, H / T\right)$ at arbitrary temperature obey[18]

$$
Z_{S}\left(i \frac{H}{T_{K}}, \frac{H}{T}\right) Z_{S}\left(-i \frac{H}{T_{K}}, \frac{H}{T}\right)=1+Z_{S+1 / 2}\left(\frac{H}{T_{K}}, \frac{H}{T}\right) Z_{S-1 / 2}\left(\frac{H}{T_{K}}, \frac{H}{T}\right)
$$

with $Z_{0} \equiv 1$. These relations hold for any $g$. However, the quantum-group algebra has the peculiar property that when $g=P / Q$ for mutually prime integers $P$ and $Q$, then $\left(S_{ \pm}\right)^{Q}=0$. Thus as opposed to ordinary $S U(2)$, the representation of spin $Q / 2$ is reducible. This means that the partition functions "truncate" when $g$ is rational (i.e. $q$ is a root of unity) [16]:

$$
Z_{Q / 2}\left(\frac{H}{T_{K}}, \frac{H}{T}\right)=Z_{Q / 2-1}\left(\frac{H}{T_{K}}, \frac{H}{T}\right)+2 \cosh \left[Q \frac{H}{2 T}\right] \quad g=\frac{P}{Q}
$$

In the subsequent analysis, this truncation will explain why the curves used to describe the magnetization have finite genus for $g<1$ rational, but infinite for $g=1$ or irrational. 
This paper will be concerned exclusively with the physics at zero temperature, so that the magnetization depends only on $H / T_{K}$ and $g$. In this limit the fusion relations (8) become linear because the free energy $F_{S}=-T \ln Z_{S}$ remains finite as $T \rightarrow 0$. In terms of the magnetization $M_{S}=-\partial F_{S} / \partial H$, the limit of (8) is

$$
M_{S}(i u)+M_{S}(-i u)=M_{S-1 / 2}(u)+M_{S+1 / 2}(u)
$$

The argument $i u$ is meant as the continuous deformation of $u \rightarrow i u$ at fixed large $|u|$. Because of the $\ln (u)$ in (7), there are non-trivial monodromies in $M_{S}(u)$ as a function of $u$, so for example $M_{S}\left(e^{2 \pi i} u\right)$ is not necessarily equal to $M_{S}(u)$. The quantum-group truncation (9) also simplifies in the zero temperature limit to

$$
M_{Q / 2}(u)=Q / 2 .
$$

These relations are the analog of the Seiberg-Witten monodromy relations at infinity.

\section{The Result}

In this section we will show that the magnetization of the spin- $S$ Kondo problem is given by

$$
M_{S}(u)=\frac{i u}{4 \pi} \int_{\mathcal{C}_{\mathcal{S}}} \frac{d x}{x y}
$$

where

$$
y^{2}=(-1)^{2 S}\left(x-x^{g}\right)+u^{2}
$$

where the contour surrounds the "first" $2 S$ branch points in a fashion to be described.

The proof of this formula follows indirectly from the integral formula (7) and the fusion relations (10). Using (7), the magnetization can be expanded in a power series around $u \rightarrow \infty$ (close to the weakly-coupled unstable fixed point) and around $u=0$ (the strongly-coupled stable fixed point), each series with a finite radius of convergence (except for $g=1$, which will be discussed in section 5). The large- $u$ series is (correcting a few typos in [6])

$$
M_{1 / 2}(u)=\frac{1}{2 \sqrt{\pi}} \sum_{n=0}^{\infty} \frac{(-1)^{n}}{n !} \frac{\Gamma\left(\frac{1}{2}+n(1-g)\right)}{\Gamma(1-n g)} u^{-2 n(1-g)}
$$

while the small- $u$ expression is

$$
M_{1 / 2}(u)=\frac{1}{\sqrt{\pi}} \sum_{n=0}^{\infty} \frac{(-1)^{n}}{n !(2 n+1)} \frac{\Gamma\left(1+(n+1 / 2) \frac{1}{(1-g)}\right)}{\Gamma\left(1+(n+1 / 2) \frac{g}{(1-g)}\right)} u^{2 n+1} .
$$


The non-trivial monodromies in the magnetization as the physical parameter $u$ is varied in the complex plane are apparent in these expansions: taking $u \rightarrow e^{2 \pi i} u$ at fixed $|u|$ in (14) does not bring the magnetization back to its original value.

The integral expression (12) follows from either expansion by using the integral representation

$$
\frac{\Gamma(a)}{\Gamma(a+b)}=\Gamma(1-b) \frac{i}{2 \pi} \int_{\mathcal{C}_{01}} d t t^{a-1}(t-1)^{b-1} .
$$

The integration contour $\mathcal{C}_{01}$ is not closed but rather starts at the origin, loops around the branch point at $t=1$, and returns to the origin. This formula is valid when $\operatorname{Re}(a)>0$ and $b$ is not an integer. Using this in (14) yields

$M_{1 / 2}(u)=\frac{i}{2 \pi^{3 / 2}} \sum_{n=0}^{\infty} \frac{(-1)^{n}}{n !} \Gamma(1 / 2+n) \int_{\mathcal{C}_{01}} d t t^{n(1-g)-1 / 2}(t-1)^{-n-1 / 2} u^{-2 n(1-g)}$.

Since the sum is absolutely convergent, we can do the sum before the integral. The sum is of the form

$$
\sum_{n=0}^{\infty} \frac{(-1)^{n}}{n !} \Gamma(1 / 2+n) z^{n}=\frac{\sqrt{\pi}}{\sqrt{1+z}}
$$

This yields

$$
M_{1 / 2}(u)=\frac{i}{4 \pi} \int_{\mathcal{C}_{0, \text { branch }}} \frac{d t}{t^{1 / 2}} \frac{1}{\left(t-1+t^{1-g} u^{-2(1-g)}\right)^{1 / 2}}
$$

where the contour $\mathcal{C}_{0, \text { branch }}$ starts at the origin, loops around the square-root branch point on the positive real $t$-axis and returns to the origin. To obtain the result (12) for $S=1 / 2$, one defines $x=u^{2} / t$ and changes variables. The magnetization for a spin-1/2 impurity is therefore

$$
M_{1 / 2}(u)=\frac{i}{4 \pi} \int_{\mathcal{C}_{1 / 2}} \frac{d x}{x} \frac{u}{\left(x^{g}-x+u^{2}\right)^{1 / 2}} .
$$

The contour $\mathcal{C}_{1 / 2}$ is displayed in figure 1 . It starts at $\infty$, loops around the square-root branch point along the positive real axis and returns to $\infty$. Such a branch point must exist for real $u$ because $y^{2}=x^{g}-x+u^{2}$ is negative for $x$ large but positive for $x=0$. For complex $u$ we define $\mathcal{C}_{1 / 2}$ as the continuous deformation of this curve away from $u$ real.

This integral expression for the magnetization is much simpler than the original result (7). This expression is analytic in $u$ except at a few singularities. It is thus an exact, non-perturbative expression for the magnetization. 


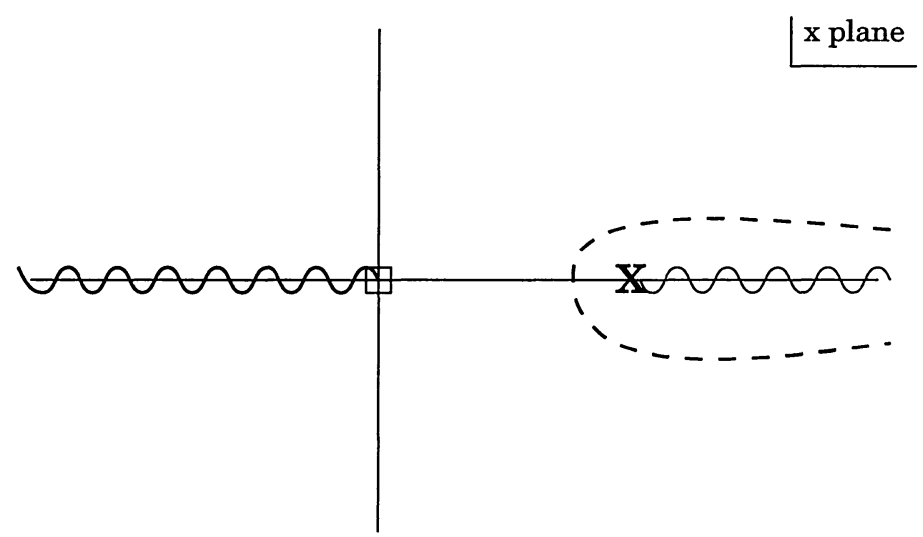

Figure 1: The dotted line is the integration contour for $M_{1 / 2}$ in the $x$-plane. The box is the simple pole, the $\mathrm{X}$ the square-root branch point on the first sheet of $x$, and the wavy lines are the square-root branch cut and the $x^{g}$ cut from the origin.

A singularity can occur at values $u=u_{j}$ where two roots coincide. This happens when both $x^{g}-x+u_{j}^{2}=0$ and $g x^{g-1}-1=0$, so

$$
u_{j}^{2}=g^{g /(1-g)}(g-1) e^{i 2 \pi j /(g-1)}
$$

where $j$ is some integer. All possible singularities are on the circle $|u|=\left|u_{j}\right|$; for $g$ irrational they are dense along this circle. There are no values of $u$ where three or more roots coincide. The integral (17) does not diverge at all of the values $u=u_{j}$, but only at values where the contour $\mathcal{C}_{1 / 2}$ goes in between the two coinciding roots. It is easy to check that this will never happen for real values of $u$ (where the couplings are "physical"). These singularities are the Lee-Yang zeroes of the partition function, but there is no obvious physical interpretation of them here like in gauge theory. In gauge theory, all complex values of $u$ are physical, and the singularities correspond to couplings where some particle becomes massless [1].

Since it is analytic for $u$ real and positive, the integral expression (17) must reproduce not only the weak-coupling series expansion (14) from which it was constructed, but also the strong-coupling series expansion (15). For $|u|<\left|u_{j}\right|$, the contour $\mathcal{C}_{1 / 2}$ can be deformed so that $\left|x-x^{g}\right|>|u|^{2}$ for all values of $x$. Expanding the square root in powers of $u^{2}$ yields

$$
M_{1 / 2}(u)=\frac{1}{4 \pi^{3 / 2}} \sum_{n=0}^{\infty} \frac{(-1)^{n}}{n !} \Gamma(1 / 2+n) \int_{\mathcal{C}_{1 / 2}} \frac{d x}{x}\left(x^{g}-x\right)^{-(n+1 / 2)} u^{2 n+1} .
$$

Defining a new variable $t=x^{g-1}$ and using the identity (16) yields the 
expansion (15). The value $u=\left|u_{j}\right|$ is therefore the value at which the perturbation expansions diverge; i.e. (14) converges for $|u|>\left|u_{j}\right|$ whereas (15) converges for $|u|<\left|u_{j}\right|$. The small- $u$ expansion is like the strongcoupling instanton expansion in gauge theory.

There are two kinds of branch points in the integrand; a square-root branch cut at every root of $x-x^{g}-u^{2}$, and another at the origin due to the $x^{g}$ term. If desired, the former can be removed by defining a new variable $t=\ln x$, leaving only a simple pole at the origin. The square-root branch points cannot be removed by a reparamaterization of $x$. They are very interesting, because the higher-spin magnetizations can be expressed by contours around these branch points.

The higher-spin magnetizations are found from the spin-1/2 magnetization by using the fusion formula (10). This requires analytically continuing the spin-1/2 magnetization into the complex- $u$ plane. How to do this is most easily seen by first studying rational values of $g$, where $g=P / Q$ for mutually prime integers $P$ and $Q$. The branch cut from the origin can be removed by defining a new variable $r=x^{Q}$, yielding

$$
M_{1 / 2}(u)=\frac{i Q}{4 \pi} \int_{\mathcal{C}_{1 / 2}} \frac{d r}{r} \frac{u}{\left(r^{P}-r^{Q}+u^{2}\right)^{1 / 2}} .
$$

When $|u|$ is large, the roots $r_{k}$ of the polynomial $r^{P}-r^{Q}+u^{2}$ are approximately

$$
r_{k} \approx e^{i 2 \pi k / Q} u^{2 / Q}
$$

for $k=0, \ldots, Q-1$. The roots $r=\tilde{r}_{k}$ of the polynomial $r^{p}-r^{Q}-u^{2}$ at large $|u|$ are

$$
\tilde{r}_{k} \approx e^{i \pi(2 k+1) / Q} u^{2 / Q}
$$

for $k=0, \ldots, Q-1$. Therefore under the continuation $u \rightarrow i u$ at fixed large $|u|$, the root $r_{k}$ moves to $\tilde{r}_{k}$, while under $u \rightarrow-i u, r_{k}$ moves to $\tilde{r}_{k-1}$. In particular, the root $r_{0}=u^{2 / Q}$ on the real axis for real $u$ moves to $|u|^{2 / Q} e^{ \pm i \pi / Q}$ under $u \rightarrow \pm i u$. The contour $\mathcal{C}_{1 / 2}$ in (19) rotates accordingly.

Now it is clear how to utilize the fusion relation (10) to obtain higher spins. The spin- 1 magnetization is

$$
\begin{aligned}
M_{1}(u) & =M_{1 / 2}(i u)+M_{1 / 2}(-i u) \\
& =\frac{i Q}{4 \pi} \int_{\mathcal{C}_{\infty \tilde{0}}} \frac{i u}{\left(r^{P}-r^{Q}-u^{2}\right)^{1 / 2}}+\frac{i Q}{4 \pi} \int_{\mathcal{C}_{\infty} \widetilde{Q-1}} \frac{-i u}{\left(r^{P}-r^{Q}-u^{2}\right)^{1 / 2}}
\end{aligned}
$$

where the contour $\mathcal{C}_{\infty \tilde{k}}$ starts at infinity and loops around the branch point at $\tilde{r}_{k}$. Up to a minus sign, the integrand is the same for both pieces, so 


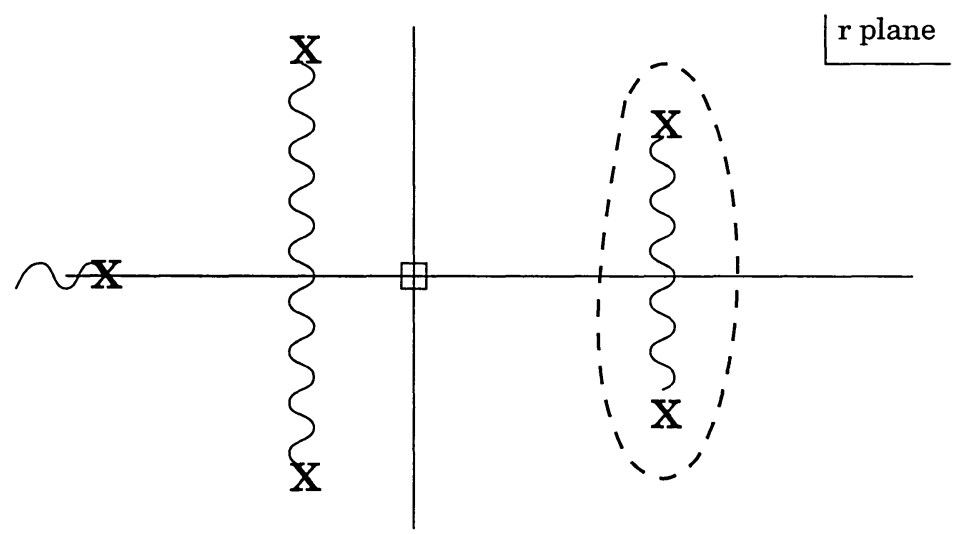

Figure 2: The dotted line is the integration contour for $M_{1}$ in the $r$ plane for $g=4 / 5$. The box is the simple pole, an X is a square-root branch point, and the wavy lines are the square-root branch cuts. This schematic drawing is for large $u$; at $u=\left|u_{j}\right|$ the two branch points on the right meet at the real axis, and for $u<\left|u_{j}\right|$ they both lie on the real axis.

the two contours can be subtracted from each other. This yields a closed contour encircling both branch points, as displayed in figure 2 .

As $u$ is decreased, the roots $\tilde{r}_{k}$ no longer obey the approximate relation (20). It follows from (18) that when $u=\left|u_{j}\right|$ these two branch points coincide at the value $x=g^{1 /(1-g)}$. The integral does not diverge because the contour is not trapped between these two roots. Since $M_{1}$ is analytic at $u=\left|u_{j}\right|$ it can be continued to $u<\left|u_{j}\right|$ where these two roots are no longer complex conjugates but are both on the real axis. The contour $\mathcal{C}_{1}$ still just loops around the two. In fact, at value $u=\left|u_{j}\right|$ where these roots coincide, the integral can be done by residue. This yields the amusing relation

$$
M_{1}\left(g^{1 /(2-2 g)}(1-g)^{1 / 2}\right)=\frac{1}{\sqrt{2 g}} .
$$

The value $u=\left|u_{j}\right|$ can be thought of as the crossover point, the limit of both the weak-coupling and strong-coupling series' applicability. It is ironic that for spin 1 , the magnetization takes on a simple value at this point.

In terms of the original integration variable $x$,

$$
M_{1}(u)=\frac{1}{4 \pi} \int_{\mathcal{C}_{1}} \frac{u}{\left(x^{g}-x-u^{2}\right)^{1 / 2}}
$$

where the contour $\mathcal{C}_{1}$ surrounds the "first" two branch points. More precisely, 
the first two branch points are those which approach $x \approx \pm i u$ for $u$ large, coincide at $u=u_{j}$, and approach 0 and 1 at $u$ small.

The magnetizations for higher spins are built up from $M_{1 / 2}$ in a similar manner. The fusion relation can be rewritten as

$$
M_{S}(u)=\sum_{j=-S+1 / 2}^{S-1 / 2} M_{1 / 2}\left(e^{i \pi j} u\right)
$$

where $M_{1 / 2}\left(e^{i \alpha} u\right)$ means $M_{1 / 2}(u)$ continued from $u \rightarrow e^{i \alpha} u$ at fixed large $|u|$. All half-integer-spin magnetizations therefore utilize the original curve $y^{2}=x^{g}-x+u^{2}$, while the integer-spin ones use $y^{2}=x^{g}-x-u^{2}$. The integration contours encircle the first $2 S$ branch points. For example, (23) gives

$$
M_{3 / 2}(u)=M_{1 / 2}(u)+M_{1 / 2}\left(e^{i \pi} u\right)+M_{1 / 2}\left(e^{-i \pi} u\right)
$$

At large $u$, continuing $u \rightarrow e^{i \pi} u$ means that the contour starts at infinity and goes around $r_{1}$. Because of the relative - sign, the contour for $M_{1 / 2}(u)+M_{1 / 2}\left(e^{i \pi} u\right)$ starts at infinity and surrounds both $r_{0}$ and $r_{1}$. Adding $M_{1 / 2}\left(e^{-i \pi} u\right)$ means that the contour surrounds $r_{Q-1}$ as well. This is illustrated in figure 3. In terms of the original variable $x$, the contour $C_{3 / 2}$ surrounds the "first" three branch points. In general, the contour $\mathcal{C}_{S}$ is the contour which surrounds the first $2 S$ branch points but does not go around the origin. This definition completes the proof of (12).

However, one remaining question must be answered. For $g$ rational, there are only $Q$ branch points. What are the "first" $2 S$ branch points when $2 S>Q$ ? Defining $x=r^{Q}$ and changing variables as in (19) yields

$$
M_{S}(u)=\frac{i Q}{4 \pi} \int_{\mathcal{C}_{S}} \frac{d r}{r} \frac{u}{\left((-1)^{2 S}\left(r^{Q}-r^{P}\right)+u^{2}\right)^{1 / 2}} .
$$

When $2 S=Q$, the contour surrounds all the square-root branch points but does not surround the simple pole at the origin as shown in figure 3. The contour can therefore be deformed to surround only the origin and none of the branch points. The integral is then easily done by residue, yielding $M_{Q / 2}=Q / 2$. This is precisely the quantum-group truncation from (11)! The fusion relations still hold for $S>Q / 2$, but all the magnetizations can be expressed in terms of lower-spin ones. Likewise, the contours $\mathcal{C}_{S}$ for $S>Q / 2$ are expressed in terms of the lower-spin contours plus the contour surrounding the origin. The latter contributes only multiples of $Q / 2$ to the magnetization.

The equation $y^{2}=(-1)^{2 S}\left(r^{Q}-r^{P}\right)+u^{2}$ defines a hyperelliptic curve, which is a Riemann surface of genus $(Q-1) / 2$ for $Q$ odd and $(Q-2) / 2$ for 


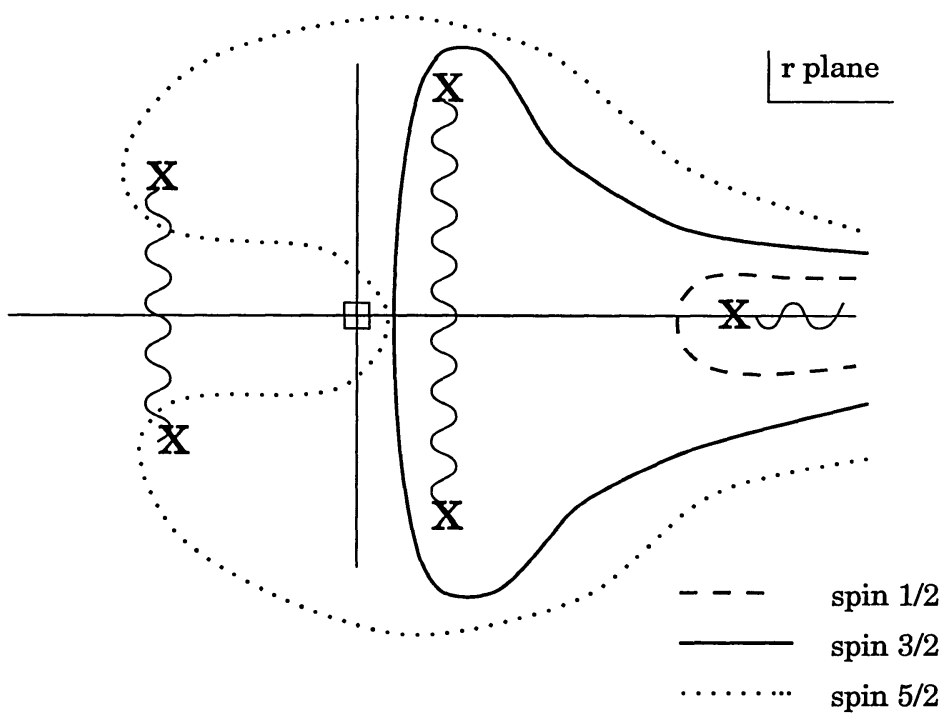

Figure 3: The contours for $M_{1 / 2}, M_{3 / 2}$ and $M_{5 / 2}$ in the $r$-plane for $g=4 / 5$. The box is the simple pole, the $\mathrm{X}$ the square-root branch point, and the wavy lines are the square-root branch cuts.

$Q$ even. The contours $\mathcal{C}_{S}$ can be decomposed into combinations of contours on the surface. Thus the quantum-group truncation allows the associated surface to be of finite genus. It seems very likely that there is a deep reason for this. However, there is one obvious puzzling question. When $g=2 / 3$ (which, perhaps not surprisingly, is where the anisotropic Kondo model is supersymmetric [19]), the surface is a torus. In this context, what is the meaning of the modular parameter $\tau$ of the torus? $M_{3 / 2}$ is trivial here, so for a fixed surface only $M_{1 / 2}$ is non-trivial ( $M_{1}$ is related to a different surface). In other words, is there any physical interpretation of the contour integral around the other cycle of the torus (the integral surrounding the branch points $r_{0}$ and $\left.r_{1}\right)$ ?

\section{$4 \quad g \rightarrow 1 / g$ Duality and The Boundary Sine-Gordon Model}

In this section I discuss the $g \rightarrow 1 / g$ duality present in the anisotropic Kondo model, and the self-duality of the boundary sine-Gordon model.

The boundary sine-Gordon model is another integrable 1+1-dimensional field theory defined on the half-line. It is deeply related to the anisotropic 
Kondo model $[16,20]$, but here there is no extra boundary degree of freedom like the impurity spin. The bulk Lagrangian is still (4), but the boundary Lagrangian is

$$
L_{B S G}=2 v \cos \phi(0) .
$$

In this model, the parameter $H / g$ is replaced by the voltage $V$, while $v$ is related to a boundary scale $T_{B}$ like $\lambda$ is related to $T_{K}$. The analog of the magnetization is the current $I$ arising from the applied voltage. The power-series expansions for the normalized current $\mathcal{I}=I / g V$ as a function of $u \propto V v^{-1 /(1-g)}$ are [4]

$$
\mathcal{I}(u, g)=1-\sum_{n=1}^{\infty} a_{n}(g) u^{2 n(g-1)}
$$

and

$$
\mathcal{I}(u, g)=\sum_{n=1}^{\infty} a_{n}(1 / g) u^{2 n(1 / g-1)}
$$

where

$$
a_{n}(g)=\frac{(-1)^{n}}{n !} \frac{\sqrt{\pi}}{2} \frac{\Gamma(n g+1)}{\Gamma(3 / 2+n(g-1))} .
$$

This model is self-dual because the weak-coupling expansion is identical to the strong-coupling expansion by the exchange $g \rightarrow 1 / g$. This leads to a simple statement about the behavior of the current as an analytic function of $g[4]$ :

$$
\mathcal{I}(u, g)=1-\mathcal{I}(u, 1 / g)
$$

A simple integral expression for the current can be derived from either expansion (26) or (27). The proof is similar to that given in section 3 for the Kondo model. The result is

$$
\mathcal{I}(u, g)=\frac{i}{4 u} \int_{\mathcal{C}_{B}} d x \frac{1}{\sqrt{x+x^{g}-u^{2}}}
$$

where the curve $\mathcal{C}_{B}$ starts at the origin, loops around the branch point on the positive real $x$-axis, and returns to the origin. Proving the selfduality relation (29) using (30) is easy because this integral expression is valid for all values of $g$ : start with $\mathcal{I}(u, 1 / g)$, change variables $x \rightarrow x^{g}$, and integrate by parts. The self-duality of the boundary sine-Gordon model and some generalizations is discussed in detail in [21]. In the context of the fractional quantum Hall effect, this duality exchanges electrons with Laughlin quasiparticles.

The $g \rightarrow 1 / g$ duality in the Kondo model is not so obvious. Nevertheless, it was noted some time ago [3] that the isotropic Kondo model has a duality 
relating the large- $u$ expansion for spin $S$ to a piece of small- $u$ expansion for spin $S+1 / 2$. The shift in $S$ is not shocking because at the strongly-coupled $(u=0)$ fixed point, one of the electrons binds to the impurity, effectively reducing the spin from $S+1 / 2$ to $S$. This duality is not a self-duality, because it maps one spin onto another. To see how this duality arises using the integral (12), first note that if one changes variables $x \rightarrow x^{1 / g}$, one has

$$
M_{S}(u)=\frac{i}{4 \pi g} \int \frac{d x}{x} \frac{u}{\left((-1)^{2 S+1}\left(x^{1 / g}-x\right)+u^{2}\right)^{1 / 2}} .
$$

where the contour must be defined. Up to the extra factor of $g$ in front, the integrand is exactly that for the magnetization with $g$ replaced with $1 / g$, and integer spins exchanged with half-integer. However, a simple formula like (29) does not instantly follow because the contour in (31) must be carefully defined.

The subtlety involving the dual contours can be seen in the small- $u$ expansion of $M_{1}(u)$. As discussed in the previous section, for $u<\left|u_{j}\right|$ the contour $\mathcal{C}_{1}$ loops around the two roots on the positive real axis. For $u$ small, these roots of $x-x^{g}+u^{2}$ are at $x \approx u^{2 / g}$ and at $x \approx 1$. The contour $\mathcal{C}_{S}$ can be decomposed into two contours: one starting at infinity and looping around the root near $x=1$, and the other starting at infinity and looping around the root near $x=u^{2 / g}$. The integral can therefore be split into two pieces. The first piece is like $M_{1 / 2}(u)$, but the integrand is slightly different because of the $(-1)^{2 S}$. For small $u$ this piece can still be expanded in powers of $u^{2 n+1}$ (the resulting coefficients are in fact $i(-1)^{n}$ times those in (15); this piece is purely imaginary). The duality is seen in the second piece, which is denoted $\widetilde{M}_{1}(u)$. Defining a new variable $t=x^{g} / u^{2}$ gives

$$
\widetilde{M}_{1}(u)=\frac{i}{4 \pi g} \int_{\mathcal{C}_{\infty 1}} \frac{d t}{t} \frac{1}{\left(u^{-2+2 / g} t^{1 / g}-t+1\right)^{1 / 2}} .
$$

The contour $\mathcal{C}_{\infty 1}$ loops around the branch point near $t=1$. For small $u$ and fixed $t$, the square root can be expanded in powers of $u^{(-2+2 / g)}$ :

$$
\widetilde{M}_{1}(u)=\frac{i}{4 \pi^{3 / 2} g} \int_{\mathcal{C}_{\infty 1}} \frac{d t}{t} \sum_{n=0}^{\infty} \frac{(-1)^{n}}{n !} \Gamma(n+1 / 2) t^{n / g}(1-t)^{-n-1 / 2} u^{2 n(1 / g-1)} .
$$

The resulting integral in $t$ converges if $n<g /(2(1-g)$ ) (or equivalently $\left.u^{2 n(1 / g-1)}<u\right)$, but the terms for higher $n$ can formally be defined by continuing in $g$ from values close to 1 . The integrals for larger $n$ diverge because integer powers $u^{n}$ (possibly multiplying $\ln (u)$ ) also contribute to the small $u$-expansion of $\widetilde{M}_{1}(u)$. (This can be seen explicitly by breaking $\mathcal{C}_{\infty 1}$ into two pieces so that one can construct convergent expansions.) The 
integer powers must cancel the imaginary pieces from the first contour to ensure that $M_{1}(u)$ is real for real positive $u$. By using the identity (16), the magnetization is

$$
M_{1}(u)=\frac{1}{2 \sqrt{\pi} g} \sum_{n=0}^{\infty} \frac{(-1)^{n}}{n !} \frac{\Gamma\left(\frac{1}{2}+n(1-1 / g)\right)}{\Gamma(1-n / g)} u^{-2 n(1-1 / g)}+\mathcal{O}\left(x^{n}\right) .
$$

The $\mathcal{O}\left(x^{n}\right)$ terms include the contributions from the first contour as well, and also include terms like $x \ln x$. The duality in the Kondo model is now apparent: the contributions to $M_{1}(u)$ at small $u$ displayed in (33) are precisely those in the large- $u$ expansion of $M_{1 / 2}(u)$ in (14), with the replacement $g \rightarrow 1 / g$, up to the extra factor of $g$. Thus for example, we recover the fact that $M_{1}(0)=1 /(2 g)$, as mentioned before.

The proof of the duality for general $S$ is straightforward. For example, the contour $\mathcal{C}_{3 / 2}$ can be split into two contours, one around the branch point on the positive real axis, and the other surrounding just the first two complex-conjugate branch points (e.g. $r_{1}$ and $r_{Q-1}$ for rational $g=$ $P / Q)$. The first contour gives merely $M_{1 / 2}(u)$. The second contour is best understood by using the variable $t=x^{g} / u^{2}$ just like in (32). In the small- $u$ limit, the second contour surrounds the square-root branch point $t=-1$ just above the branch cut from the origin and and the square-root branch point $t=-1$ just below the branch cut from the origin. This contour is the same as the contour for $M_{1}(u)$ in the large- $u$ limit, and moreover, the integrand is the same as $M_{1}(u)$ for large $u$, up to the replacement $g \rightarrow 1 / g$ and with an overall $1 / g$. In other words,

$$
\begin{aligned}
M_{3 / 2}(u) & =\frac{i}{4 \pi} \int_{\mathcal{C}_{3 / 2}} \frac{d x}{x} \frac{u}{\left(x^{g}-x+u^{2}\right)^{1 / 2}} \\
& =M_{1 / 2}(u)+\frac{i}{4 \pi g} \int_{\mathcal{C}_{1}} \frac{d t}{t} \frac{1}{\left(t-u^{-2+2 / g} t^{1 / g}+1\right)^{1 / 2}} \\
& =M_{1 / 2}(u)+\left.\frac{1}{g} M_{1}(u)\right|_{g \rightarrow 1 / g}
\end{aligned}
$$

By this argument, for integer $S$ one has

$$
M_{S+1 / 2}(u)=M_{1 / 2}(u)+\left.\frac{1}{g} M_{S}(u)\right|_{g \rightarrow 1 / g}
$$

where the $g \rightarrow 1 / g$ in the last line is precisely defined at least at small $u$. For half-integer $S$, such a simple relation does not seem to be true, but by these arguments

$$
M_{S+1 / 2}(u)=\left.\frac{1}{g} M_{S}(u)\right|_{g \rightarrow 1 / g}+\mathcal{O}\left(u^{n}\right)
$$


is true for all $S$. This is the Kondo duality discussed in [3]. Given the integral relation (31) it seems likely that this is not the only form of $g \rightarrow 1 / g$ duality in the Kondo model.

\section{The SU(2) Point}

A simple integral expression for the zero-temperature magnetization of the anisotropic Kondo model was derived in the section 3 . The results most resemble the Seiberg-Witten results for gauge theory when $g$ is rational and less than 1, and the integral is on a finite-genus hyperelliptic curve. However, the model which is most like QCD is the $S U(2)$-symmetric case $g=1$. As explained in section 2, the Kondo model is asymptotically free, develops a mass scale even though all parameters are naively dimensionless, and exhibits a crossover into a strong-coupling phase. In this section I discuss the isotropic Kondo model in more detail, find explicit expressions for its perturbative coefficients, and highlight its $g \rightarrow 1 / g$ duality.

Taking the limit $g \rightarrow 1$ of (12) yields the appropriate integral expression. The definition (6) shows that for fixed $H / T_{K}, u^{2} \rightarrow(1-g) H^{2} /\left(2 \pi T_{K}^{2}\right)$ as $g \rightarrow 1$, so

$$
\lim _{g \rightarrow 1} x^{g}-x+u^{2}=(g-1)\left(x \ln x-H^{2} /\left(2 \pi T_{K}^{2}\right)\right) .
$$

Denoting $\mathcal{M}_{S}\left(H / T_{K}\right)=\lim _{g \rightarrow 1} M_{S}(u)$, its integral form is

$$
\mathcal{M}_{S}\left(H / T_{K}\right)=\frac{i}{4 \pi} \int_{\mathcal{C}_{S}} \frac{d x}{x} \frac{H / T_{K}}{\left((-1)^{2 S} 2 \pi x \ln x+\left(H / T_{K}\right)^{2}\right)^{1 / 2}}
$$

The contour $\mathcal{C}_{S}$ is defined as in the previous section; there are of course an infinite number of solutions to the transcendental equation $2 \pi x \ln x=$ $\pm\left(H / T_{K}\right)^{2}$, so as expected for $S U(2)$ symmetry, all the $\mathcal{M}_{S}$ are independent.

Weak- and strong-coupling perturbative expansions can be derived from (37), but there are some subtleties. For spin $1 / 2$, the small $H / T_{K}$ expansion follows as before, by expanding the square root in powers of $\left(H / T_{K}\right)^{2}$. One indeed obtains the $g \rightarrow 1$ limit of (15). However, the form of the large- $H / T_{K}$ expansion (which corresponds to the weak-coupling expansion in terms of the original coupling $\lambda$ ) is not so obvious: the coefficients in (14) diverge as $g \rightarrow 1$, while the powers all collapse to zero. As seen from the definition (2) of $T_{K}$ at the $S U(2)$ point, the perturbative expansion in $\lambda$ should include terms with $\log T_{K}$. This is the usual behavior of an asymptotically-free theory. This suggests defining a renormalized coupling $z(H, \lambda, D)(D$ is the 
cutoff) which is renormalization-group invariant and which obeys $z \approx \lambda$ for $\lambda \ll 1$. The explicit perturbative results (summarized in [6]) suggest

$$
\ln \left(H / T_{K}\right)=\frac{1}{z}-\frac{1}{2} \ln (z / 4 \pi)
$$

This differs from the definition in [6] by a $z$-independent numerical factor on the right; the reason will be apparent shortly.

Weak coupling $\left(H / T_{K}\right.$ large) is equivalent to $z$ small. The magnetization $M_{1 / 2}(u)$ can be expanded in a power series in $z$ :

$$
\begin{aligned}
\mathcal{M}_{1 / 2}(z) & =\frac{i}{4 \pi} \int_{\mathcal{C}_{1 / 2}} \frac{d x}{x} \frac{\sqrt{2}}{\left(-x\left(\ln x+\ln \left(H^{2} / 4 \pi T_{K}^{2}\right)\right)+2\right)^{1 / 2}} \\
& =\frac{i}{4 \pi} \int_{\mathcal{C}_{1 / 2}} \frac{d x}{x} \frac{\sqrt{2}}{(-x(\ln x+2 / z-\ln z)+2)^{1 / 2}} \\
& =\frac{i}{4 \pi} \int_{\mathcal{C}_{1 / 2}} \frac{d x}{x} \frac{1}{(-(z / 2) x \ln x-x+1)^{1 / 2}}
\end{aligned}
$$

where the first line required a rescaling $x \rightarrow x H^{2} /\left(4 \pi T_{K}^{2}\right)$ and the third line required $x \rightarrow z x$. Now the square root can be expanded in powers of $z$ :

$$
\mathcal{M}_{1 / 2}(z)=\sum_{n=0}^{\infty} \mathcal{A}_{n} z^{n}
$$

where

$$
\mathcal{A}_{n}=\frac{(-1)^{n}}{2^{n+1} \pi^{3 / 2} n !} \Gamma(n+1 / 2) \int_{1}^{\infty} d x x^{n-1}(\ln x)^{n}(x-1)^{-n-1 / 2} .
$$

These integrals converge for all $n$, but with a different choice of the constant in (38) they would not. This expansion in $z$ is asymptotic: for large enough $x$ the term $z x \ln x$ eventually dominates the other terms in (39) no matter how small $z$ is, so the expansion in powers of $z$ has zero radius of convergence. Of course this is expected for a theory with dimensional transmutation; what is remarkable here is that even the non-perturbative corrections are included in the Bethe ansatz solution of the Kondo problem and in the simple integral expression (39). The leading term is $\mathcal{A}_{0}=1 / 2$ as required, and $\mathcal{A}_{1}=-1 / 4$. The integral cannot be done in closed form for higher $n$, although it can be expressed in term of a hypergeometric function at fixed argument. Numerically, $\mathcal{A}_{2}=0.298286794, \mathcal{A}_{3}=-0.648160191, \ldots$, and as $n \rightarrow \infty, \mathcal{A}_{n} \propto n !$.

The last remaining issue is how the duality manifests itself at the $S U(2)$ point $g=1$. To find a perturbative expansion for $\mathcal{M}_{1}$ for small $H / T_{K}$, it is 
convenient to define the parameter $\tilde{z}$ analogously to $z$ :

$$
\ln \left(H / T_{K}\right)=-\frac{1}{\tilde{z}}-\frac{1}{2} \ln (\tilde{z} / 4 \pi) .
$$

The changed sign in (41) means that $\tilde{z} \rightarrow 0$ as $H / T_{K} \rightarrow 0$ (notice that $\tilde{z}$ is imaginary for large $\left.H / T_{K}\right)$. The spin-1 magnetization is then

$$
\mathcal{M}_{1}(\tilde{z})=\frac{i}{4 \pi} \int_{\mathcal{C}_{1}} \frac{d x}{x} \frac{1}{((\tilde{z} / 2) x \ln x-x+1)^{1 / 2}}
$$

For $z$ small the contour surrounds the branch points at $x \approx 2 / z$ and $x \approx 1$. As for $g<1$, the contour can be split into two pieces, the first extending from infinity around the branch point near $2 / z$, and the second extending from infinity around the branch point near 1 . The first can be expanded in a power series in $\left(H / T_{K}\right)^{n}$, as can easily be seen by returning to the original integral expression. Denoting the second piece $\widetilde{\mathcal{M}}_{1}(\tilde{z})$, it follows immediately that

$$
\widetilde{\mathcal{M}}_{1}(\tilde{z})=\mathcal{M}_{1 / 2}(-z)
$$

The expansion of $\widetilde{\mathcal{M}_{\infty}}(\tilde{z})$ in powers of $\tilde{z}$ is asymptotic. Putting the two contours back together gives

$$
\mathcal{M}_{1}(\tilde{z})=\mathcal{M}_{1 / 2}(-z)+\mathcal{O}\left(\left(H / T_{K}\right)\right) .
$$

In this sense the parameter $\tilde{z}$ is dual to $z$, just like the earlier Kondo duality required sending $g \rightarrow 1 / g$. It is possible that there is a better definition of $z$ and $\tilde{z}$ which makes the duality relation more transparent.

\section{Conclusions and Questions}

I have shown that the zero-temperature magnetization in the spin- $S$ Kondo model can be written as integrals around cycles of a curve $y^{2}=(-1)^{2 S}(x-$ $\left.x^{g}\right)+u^{2}$. At the $S U(2)$ point, this curve reduces to $y^{2}=x \ln x-H^{2} / 2 \pi T_{K}^{2}$. At rational $g<1$, this curve is of finite genus, a fact connected to the quantum-group symmetry of the model. The curve also gives a simple way to see the duality in the Kondo problem between the weak-coupling spin- $S$ problem and the strong-coupling spin- $(S+1 / 2)$ problem.

The Kondo model is integrable, which implies the existence of an infinitedimensional symmetry. The results in this paper clearly utilize the integrability of the model, so even though the Kondo model is not supersymmetric in general it does possess a great deal of symmetry. In fact, the infinitedimensional quantum-group symmetry underlying some integrable models 
can be shown to be an extension and deformation of two-dimensional $N=2$ supersymmetry (which exists at the point $g=2 / 3$ here) [22]. The question is then if such a deformation is possible in four dimensions: for example, can the $S U(2)$ gauge symmetry of [1] be deformed into some sort of quantumgroup-like symmetry, which may break the supersymmetry but still enable the Seiberg-Witten computations? In fact, by deforming away from a supersymmetric point, it is known that duality holds in non-supersymmetric string theories [23].

A potentially related issue is the fact that the results of $[1,2]$ are known to be intimately connected to classical integrable systems [24]. The models discussed in this paper are quantum integrable systems. There is no obvious relation between the two, but it is hard to imagine that there is none.

Since the Seiberg-Witten results follow from a finite-dimensional symmetry algebra, this raises a question for the two-dimensional models: is the full power of integrability necessary for these computations to be valid, or is some smaller symmetry algebra sufficient? For example, in two dimensions at least one way of exploiting a finite-dimensional symmetry algebra is known, the topological-antitopological fusion of [25]. The results here hint that there may be a completely new way of approaching $1+1$-dimensional field theories, integrable or not. One way of approaching the problem is to find a physical interpretation of the monodromies around the singularities; the fusion relation (10) says something about the monodromies at infinity, but not much about those at $|u|=\left|u_{j}\right|$. Of course, since the exact solution is known these monodromies can be found, but it would be much more desirable to know them a priori instead of a posteriori.

Another important direction to explore is to understand if these results can be extended to finite temperature, where the fusion relations (10) are nonlinear. This may seem to destroy the whole picture, since the addition of contours is a linear relation. However, the current in the boundary sineGordon model is also related to the magnetization in Kondo by a non-linear fusion relation $[4,20]$, and still obeys the integral expression discussed in section 4 and in [21].

The Kondo model is one of the grand old problems of quantum statistical mechanics. It was recognized long ago to be a useful toy model for QCD [12]. It is remarkable that more than two decades later, it still holds a few surprises.

I would like to thank K. Intriligator and P. Arnold for many useful conversations on duality, B. McCoy for interesting comments on the paper, and 
H. Saleur for related collaboration.

\section{References}

[1] N. Seiberg and E. Witten, Nucl. Phys. B426 (1994) 19; Nucl. Phys. B431 (1994) 484

[2] K. Intriligator and N. Seiberg, hep-th/9509066; W. Lerche, hepth/9611190; M.E. Peskin, hep-th/9702094; L. Alvarez-Gaume and F. Zamora, hep-th/9709180

[3] V. Fateev and P.B. Wiegmann, Phys. Lett. 81A (1981) 179

[4] P. Fendley, A.W.W. Ludwig and H. Saleur, Phys. Rev. B52 (1995) 8934, cond-mat/9503172

[5] C. Monoten and D. Olive, Phys. Lett. 72B (1977) 117; P. Goddard, J. Nuyts and D. Olive, Nucl. Phys. B125 (1977) 1; J. Cardy and E. Rabinovici, Nucl. Phys. B205 (1982) 1; J. Cardy, Nucl. Phys. B205 (1982) 17; A. Shapere and F. Wilczek, Nucl. Phys. B320 (1989) 669

[6] A.M. Tsvelick and P.B. Wiegmann, Adv. Phys. 32 (1983) 453

[7] N. Andrei, K. Furuya and J. Lowenstein, Rev. Mod. Phys. 55 (1983) 331

[8] X.G. Wen, Phys. Rev. B41 (1990) 12838; Phys. Rev. B43 (1991) 11025; C.L. Kane and M.P.A. Fisher, Phys. Rev. B46 (1992) 15233; K. Moon, H. Yi, C.L. Kane, S.M. Girvin and M.P.A. Fisher, Phys. Rev. Lett. 71 (1993) 4381, cond-mat/9304010; P. Fendley, A.W.W. Ludwig and H. Saleur, Phys. Rev. Lett. 74 (1995) 3005, cond-mat/9408068

[9] I. Affleck, Nucl. Phys. B336 (1990) 517

[10] J. Kondo, Prog. Th. Phys. 32 (1964) 37.

[11] P. Anderson, J. Phys. C3 (1970) 2436; P. Nozières, J. Low Temp. Phys. 17 (1974) 31

[12] K. Wilson, Rev. Mod. Phys. 47 (1975) 773.

[13] A.J. Leggett, S. Chakravarty, A.T. Dorsey, M.P.A. Fisher, A. Garg and W. Zwerger, Rev. Mod. Phys. 59 (1987) 1 
[14] P.B. Wiegmann, J. Phys. C24 (1981) 1463

[15] K.-D. Schotte, Z. Physik 230 (1970) 99.

[16] P. Fendley, F. Lesage and H. Saleur, J. Stat. Phys. 85 (1996) 211, condmat/9510055

[17] P. Kulish, N. Reshetikhin and E. Sklyanin, Lett. Math. Phys. 5 (1981) 393

[18] V.V. Bazhanov, S. Lukyanov and A. B. Zamolodchikov, Comm. Math. Phys. 177 (1996) 381, hep-th/9412229

[19] N.P. Warner, Nucl. Phys. B450 (1995) 663, hep-th/9506064

[20] V.V. Bazhanov, S. Lukyanov and A.B. Zamolodchikov, Comm. Math. Phys. 190 (1997) 247, hep-th/9604044

[21] P. Fendley and H. Saleur, "Self-duality in quantum impurity problems", cond-mat/9804173

[22] D. Bernard and A. LeClair, Comm. Math. Phys. 142 (1991) 99

[23] J.D. Blum and K.R. Dienes, Phys. Lett. B414 (1997) 260, hepth/9707148; Nucl. Phys. B516 (1998) 83, hep-th/9707160

[24] A. Gorsky, I. Krichever, A. Marshakov, A. Mironov and A. Morozov, Phys. Lett. B355 (1995) 466, hep-th/9505035; E. Martinec and N. Warner, Nucl.Phys. B459 (1996) 97, hep-th/9509161; for a recent review see A.Marshakov, hep-th/9709001.

[25] S. Cecotti and C. Vafa, Nucl. Phys. B367 (1991) 359; S. Cecotti, P. Fendley, K. Intriligator and C. Vafa, Nucl. Phys. B 386 (1992) 405, hep-th/9204102 